Supporting Information

\title{
Sequencing of Phosphoramidate Oligonucleotides by Acid Hydrolysis and Mass Spectrometry
}

Soheil Pourshahian* and Sergei M. Gryaznov

Janssen Pharmaceutical Companies of Johnson \& Johnson, South San Francisco, CA 94080

*E-mail: spoursha@its.jnj.com

Figure S1. Deconvoluted mass spectra of hydrolyzed ON-7.

Figure S2. Deconvoluted mass spectra of hydrolyzed ON-8.

Figure S3. Deconvoluted mass spectra of hydrolyzed ON-9. 
Figure S1. Deconvoluted mass spectra of hydrolyzed ON-7. Products from $5^{\prime}-(\times)$ and $3^{\prime}$-end $(\bullet)$ are labeled. Products labeled with $(\bullet)$ have a $5^{\prime}$-phosphate. Masses and sequences of the detected products are shown in the table. The 5'phosphate is shown as "p".

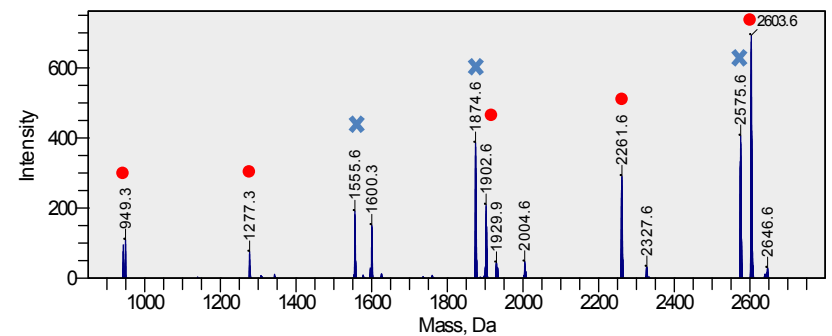

Obs. M ass Cal. M ass $5^{\prime}$

Sequence

3' Cal. Mass Obs. M ass
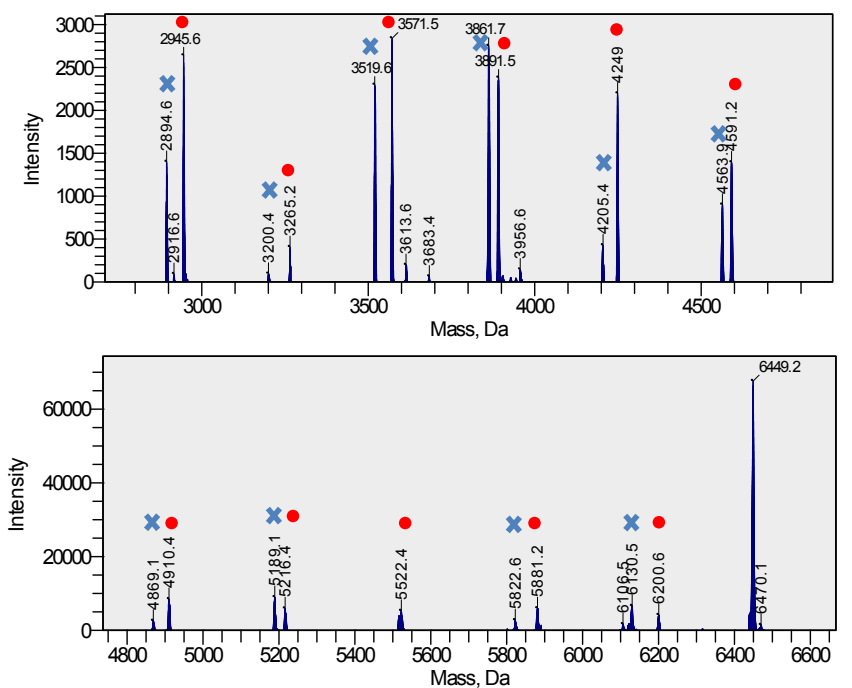

\begin{tabular}{|c|c|c|c|c|}
\hline 266.3 & pUGC CUAGUCUAA & GCUACCU & 6200.2 & 6200.6 \\
\hline 585.5 & pGC CUAGUCUAA & GCUACCU & 5881.0 & 5881.2 \\
\hline 943.7 & pCCUAGUCUAA & GCUACCU & 5522.7 & 5522.4 \\
\hline 1249.9 & pCUAGUCUAA & GCUACCU & 5216.5 & 5216.4 \\
\hline 1556.1 & pUAGUCUAA & GCUACCU & 4910.3 & 4910.4 \\
\hline 1875.3 & pAGUCUAA & GCUACCU & 4591.1 & 4591.2 \\
\hline 2217.6 & AUGCCUA & GCUACCU & 4248.9 & 4249.0 \\
\hline 2575.8 & AUGCCUAG & GCUACCU & 3890.6 & 3891.5 \\
\hline 2895.0 & AUGCCUAGU & GCUACCU & 3571.4 & 3571.5 \\
\hline 3201.2 & AUGCCUAGUC & GCUACCU & 3265.2 & 3265.2 \\
\hline 3520.4 & AUGCCUAGUCU & GCUACCU & 2946.0 & 2945.6 \\
\hline 3862.7 & AUGCCUAGUCUA & GCUACCU & 2603.8 & 2603.6 \\
\hline 4204.9 & AUGCCUAGUCUAA & oGCUACCU & 2261.5 & 2261.6 \\
\hline 4563.2 & AUGCCUAGUCUAAG & pCUACCU & 1903.3 & 1902.6 \\
\hline 4869.4 & AUGCCUAGUCUAAGC & pUACCU & 1597.1 & - \\
\hline 5188.6 & AUGCCUAGUCUAAGCU & pACCU & 1277.9 & 1277.3 \\
\hline 5516.8 & AUGCCUAGUCUAAGCUA & $\mathrm{pCCU}$ & 949.6 & 949.3 \\
\hline 5823.0 & AUGCCUAGUCUAAGCUAC & $\mathrm{pC} U$ & 643.4 & - \\
\hline 6129.2 & AUGCCUAGUCUAAGCUAC & $\mathrm{pU}$ & 337.2 & - \\
\hline
\end{tabular}


Figure S2. Deconvoluted mass spectra of hydrolyzed ON-8. Products from $5^{\prime}-(x)$ and $3^{\prime}$-end $(\bullet)$ are labeled. Products labeled with $(\bullet)$ have a $5^{\prime}$-phosphate. Masses and sequences of the detected products are shown in the table. The 5'phosphate is shown as " $\mathrm{p}$ ".

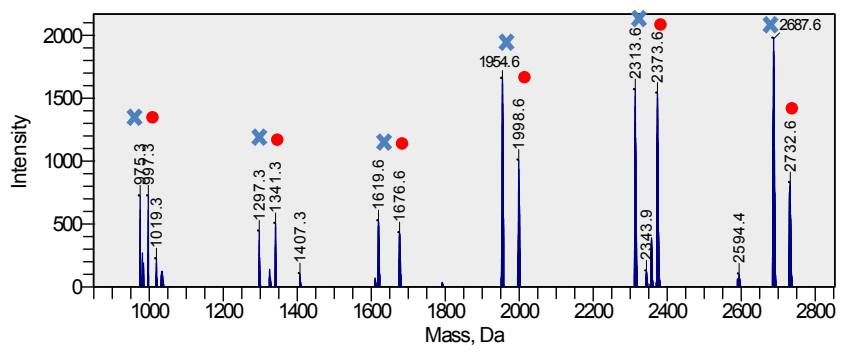

Obs. M ass Cal. Mass 5

Sequence

3' Cal. Mass Obs. Mass


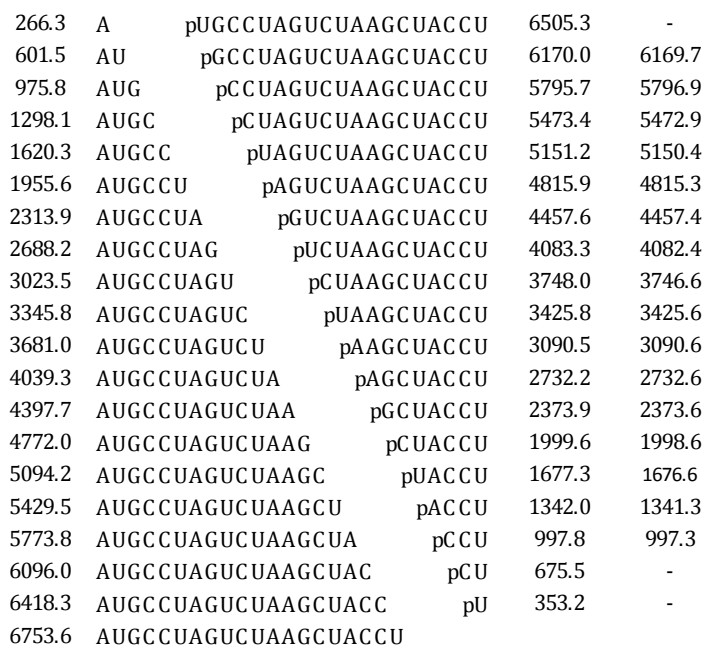


Figure S3. Deconvoluted mass spectra of hydrolyzed ON-9. Products from $5^{\prime}-(x)$ and $3^{\prime}$-end $(\bullet)$ are labeled. Products labeled with $(\bullet)$ have a $5^{\prime}$-phosphate. Masses and sequences of the detected products are shown in the table. The $5^{\prime}$ phosphate is shown as " $\mathrm{p}$ ".

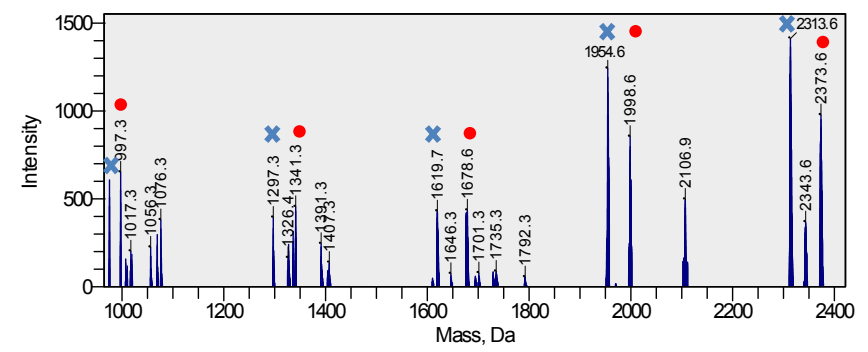

Obs. M ass Cal. Mass 5

Sequence

3' Cal. Mass Obs. Mass

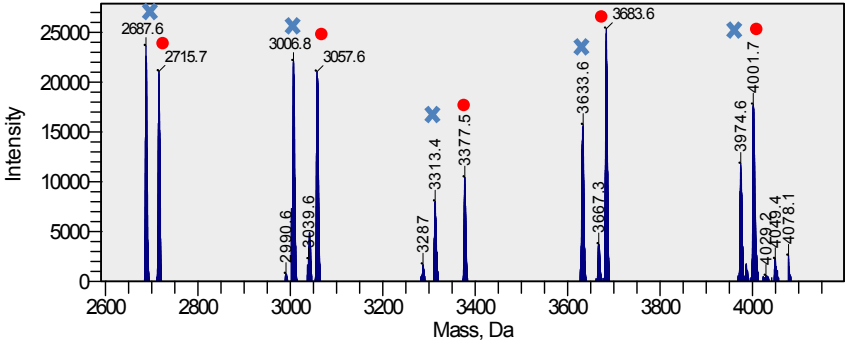

- $\quad 266.3$

A

pUGCCUAGUCUAAGCUACCU $\quad 6425.0$

AU pGCCUAGUCUAAGCUACCU 6089.7

- $\quad 601.5$

AUG pCCUAGUCUAAGCUACCU

$1297.3 \quad 1298.1$

$1619.7 \quad 1620.3$

$1954.6 \quad 1955.6$

$2313.6 \quad 2313.9$

$2687.6 \quad 2688.2$

$3006.8 \quad 3007.5$

$3313.4 \quad 3313.6$

$3633.6 \quad 3632.9$

$3974.6 \quad 3975.1$

$4317.2 \quad 4317.4$

$4691.4 \quad 4691.7$

$5014.3 \quad 5013.9$

$5348.7 \quad 5349.2$

$5693.4 \quad 5693.5$

pC UAGUCUAAGCUACCU

pUAGUCUAAGCUACCU PAGUCUAAGCUACCU

AUGCCU

pGUCUAAGCUACCU

AUGCCUA

pUCUAAGCUACCU

pCUAAGCUACCU

AUGCCUAG

pUAAGCUACCU

AUGCCUAGUC

pAAGCUACCU

AUGCCUAGUCU

pAGCUACCU

CUAGUCUA

pGCUACCU

pCUACCU

AUGC CUAGUC UAAG

pUACCU

AUGCCUAGUCUAAGC

AUGCCUAGUCUAAGCU

pACCU 1342.0

AUGCCUAGUCUAAGCUA

pCCU 997.8

AUGCCUAGUCUAAGCUAC

pCU $\quad 675.5$

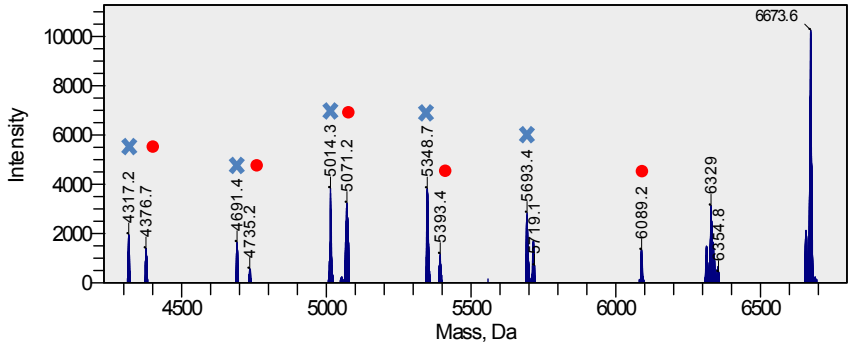

$\begin{array}{cr}- & 6338.0 \\ 6673.6 & 6673.2\end{array}$

AUGCCUAGUCUAAGCUACC

pU 353.2

-
6089.
-
5393.
5071.
4735.
4376.
4001.
3683.
3377.
3057.
2715.
2373.
1998.
1678.
1341.
997.3
-
- 